\title{
Improvement in Performance of Cold-drawn SCM435 Alloy Steel Wires through Optimization of Intercritical Annealing Parameters
}

\author{
Chih-Cheng Yang ${ }^{1 *}$ and Nan-Hua $\mathrm{Lu}^{2}$ \\ ${ }^{1}$ Department of Mechanical Engineering and Automation Engineering, Kao Yuan University, \\ 1821 Chung-Shan Road, Lu-Chu, Kaohsiung 82151, Taiwan, R.O.C. \\ ${ }^{2}$ Graduate School of Fasteners Industry Technology, Kao Yuan University, \\ 1821 Chung-Shan Road, Lu-Chu, Kaohsiung 82151, Taiwan, R.O.C.
}

(Received June 15, 2019; accepted September 19, 2019)

Keywords: intercritical annealing, formability, Taguchi method, tensile strength, ductility

The cold-forging quality alloy steel wire is generally fabricated by drawing, followed by intercritical annealing treatment to achieve the necessary formability for cold forming. The quality of a spheroidized annealing wire affects the forming quality of fasteners. The Taguchi method along with the analysis of variance (ANOVA) is used to obtain optimal intercritical annealing parameters to improve the performance of cold-drawn SCM435 alloy steel wires. The spheroidized annealing qualities of alloy steel wires are affected by various factors, such as preheating rate, spheroidized annealing temperature, prolonged heating time, holding temperature and time, low cooling rate, and temperature. The spheroidized annealing conditions affect the quality characteristics of alloy steel wires, such as tensile strength, hardness, and ductility. A series of experimental tests on cold-drawn SCM435 alloy steel wires is carried out in a commercial bell furnace with protective hydrogen atmosphere. The temperatures are precisely controlled within $\pm 2{ }^{\circ} \mathrm{C}$ and a closed-loop atmosphere control system is applied to determine the optimal atmosphere flow settings by using a zirconium oxide sensor in conjunction with annealing process equilibrium. It is experimentally revealed that the spheroidized annealing temperature, prolonged heating time, and holding temperature significantly affect the quality of annealed cold-drawn SCM435 alloy steel wires. The optimal combination of intercritical process parameters leads to obtaining the optimal mean tensile strength of $527.1 \mathrm{MPa}$, the optimal mean hardness of $76.2 \mathrm{HRB}$, and the optimal mean ductility of 0.437 with a shorter processing time. The variations of the properties are significantly reduced to achieve a much even quality for annealed wires. The new spheroidizing parameter settings evidently improve the performance measures over their values at the original settings. The performance of cold-drawn SCM435 alloy steel wires is substantially improved.

\section{Introduction}

SCM435 alloy steel wires are widely used for manufacturing mechanical parts, such as highstrength bolts and machine parts. These mechanical parts are fabricated into various shapes 
by cold forging. Sufficient formability is essential to impose a large plastic deformation on the wires during forging processes. The wires are usually produced by drawing, followed by spheroidized annealing to achieve the needed formability for cold forming. The spheroidized microstructure of steels is the most stable and well known to bring about good ductility. The majority of spheroidizing activities is to improve the ductility of steel for cold formability. ${ }^{(1)}$ The subcritical and intercritical processes are often used for the spheroidized annealing of steel wires. ${ }^{(2,3)}$ The subcritical annealing process is simply heating the steel wires to a temperature below the $\mathrm{Ac}_{1}$ temperature and maintaining at this temperature. The intercritical annealing process involves heating to, and maintaining at, a temperature between the $\mathrm{Ac}_{1}$ and $\mathrm{Ac}_{3}$ temperatures to obtain partial austenitisation, followed by slow cooling or retaining at a temperature below the critical temperature. In this study, we will focus on the intercritical annealing with a protective atmosphere of hydrogen ${ }^{(4,5)}$ because of superior operations with regard to productivity, improved mechanical properties, and surface and overall product quality. ${ }^{(6,7)}$

Many studies have been conducted to improve the spheroidization of steels. Ikpeseni et $a l .{ }^{(8)}$ investigated the effect of intercritical annealing temperature on the microstructure and mechanical properties of a $0.23 \% \mathrm{C}$ low-alloy steel. The results showed that all the evaluated mechanical properties were improved by intercritical annealing, with the samples treated at $790{ }^{\circ} \mathrm{C}$ exhibiting the optimal combination of properties. Mun and $\mathrm{Kang}^{(9)}$ implemented one full annealing cycle and two spheroidized annealing cycles to improve the forgeability of the SNCM220 alloy steel and indicated that the proposed intercritical spheroidizing heat treatment cycle was superior to the full and subcritical annealing cycles in the cold forging of SNCM220. Yang and $\mathrm{Liu}^{(10)}$ carried out a series of experimental tests on AISI 1022 low-carbon steel wires in a commercial pit-type annealing furnace with protective nitrogen atmosphere and used the Taguchi method to obtain optimal subcritical spheroidized annealing conditions to improve the mechanical properties of steel wires for cold forming. The results showed that the spheroidized annealing temperature and prolonged heating time had the greatest effect on the mechanical properties of steel wires. The optimal mean tensile strength is $384.7 \mathrm{MPa}$ and the optimal mean hardness is $129.0 \mathrm{HV}$. Yang and Wang ${ }^{(11)}$ experimentally studied AISI 10B21 steel wires also by using the Taguchi method to obtain optimal subcritical annealing conditions to improve the mechanical properties of steel wires for cold forming. It was experimentally revealed that the spheroidized annealing temperature and prolonged heating time were significant factors and that the optimal mean tensile strength of $388.6 \mathrm{MPa}$ and the optimal mean ductility of 0.418 were obtained.

Hernāndez-Silva et al. ${ }^{(12)}$ studied the spheroidization of cementite at subcritical and intercritical temperatures by evaluating a shape factor for cementite particles. It was found that previous cold deformation markedly increased the spheroidization rate of pearlitic-ferritic microstructures in AISI 1541 steel, and the spheroidization of nondeformed specimens was even faster than that observed during the subcritical annealing of cold-worked specimens. O'Brien and Hosford ${ }^{(13)}$ investigated the spheroidization of the medium-carbon steels AISI 1541 and AISI 4037 used in the bolt industry with intercritical and subcritical cycles, and indicated that the subcritical annealing could require a shorter process time than the intercritical annealing for achieving higher formability for the medium-carbon steel. Karadeniz ${ }^{(14)}$ investigated 
the subcritical spheroidization of the medium-carbon steel (AISI 4140) used in the forging industry and concluded that, initially, hardening and then the subcritical spheroidization method application give greater forgeability values in a short time. Ko et al. ${ }^{(15)}$ proposed a method of continuous shear drawing for industrial applications to medium-carbon steel wire manufacturing and revealed that the proposed method was effective in deforming pearlite colonies for easy spheroidization with subcritical annealing treatment.

By using the Taguchi robust design at the subcritical temperature of $700{ }^{\circ} \mathrm{C}$, Kamyabi-Gol and Sheikh-Amiri ${ }^{(16)}$ investigated the amount of spheroidized cementite in CK60 steel using two parameters, namely, initial microstructure and spheroidizing time. It was revealed that spheroidizing time had a larger effect on the percentage of spheroidized cementite, and better mechanical properties were expected from specimens with an unstable initial microstructure. Arruabarrena et al. $^{(17)}$ carried out a spheroidization kinetic study in a low-alloy mediumcarbon steel by image analysis techniques and indicated that the deformation accelerated spheroidization kinetics and led to a higher spheroidization degree. Ji et al. ${ }^{(18)}$ investigated the effects of subcritical annealing temperature on the microstructure and mechanical properties of SCM435 steel at soaking temperatures of $660-745{ }^{\circ} \mathrm{C}$ for $5 \mathrm{~h}$. The results showed that the subcritical annealing temperature could practically be set as $700{ }^{\circ} \mathrm{C}$, which led to obtaining a tensile strength of $625.18 \mathrm{MPa}$, a ductility of 0.232 , and a hardness lower than $185 \mathrm{HV}$ (90 HRB), and the applicability and stability of subcritical annealing were guaranteed in industrial application. Joo et al. ${ }^{(19)}$ investigated the effect of a noncircular drawing sequence on the spheroidization behavior of a medium-carbon steel wire and demonstrated that the noncircular drawing sequence might be efficient in manufacturing medium-carbon steel wires with improved spheroidization and cold workability by subcritical annealing. Okonogi and Yamazaki ${ }^{(20)}$ investigated the effects of microstructure on the mechanical properties of spheroidized annealing medium-carbon steel wire rods and the control methods of the microstructure of JIS SWRCH40K medium-carbon steel wire rods. The results indicated that in the microstructure, obtained by isothermal transformation treatment, wherein the ferrite grain size is large and the cementite is fine, lower strength and higher ductility are compatible and the formability is improved. Yang and $\mathrm{Lu}^{(21)}$ conducted a series of experimental tests on SCM435 alloy medium-carbon steel wires in a commercial hydrogen bell furnace and used the Taguchi method along with the analysis of variance (ANOVA) to obtain optimal intercritical annealing conditions to improve the mechanical properties of alloy steel wires for cold forming. It was indicated that the area reduction ratio, spheroidized annealing temperature, holding temperature, and cooling temperature significantly affect the quality of annealed SCM435 alloy steel wires. The optimal combination of process parameters leads to obtaining the optimal mean tensile strength of $567.8 \mathrm{MPa}$ and the mean ductility of 0.361 with no prior cold drawing.

For wire manufacturers, the intercritical process is used for spheroidized annealed colddrawn SCM435 alloy medium-carbon steel wires. The quality of a spheroidized annealed wire affects the forming quality of screws. To improve the annealing quality of cold-drawn SCM435 alloy steel wires, the Taguchi robust design method is used, which can determine the experimental condition having the least variability as the optimal condition. ${ }^{(22,23)}$ Various parameters affect the quality characteristics of intercritical annealing, such as preheating 
rate, spheroidized annealing temperature, prolonged heating time, holding temperature and time, cooling rate, and temperature. The spheroidized annealing parameters affect the quality characteristics of wires, such as tensile strength, ductility, and hardness. In this study, a series of experimental tests on cold-drawn SCM435 alloy steel wires is carried out in a commercial bell furnace with protective hydrogen atmosphere under completely computerized control, and the Taguchi method is used to obtain optimal intercritical annealing conditions to improve the mechanical properties of alloy steel wires for cold forming.

\section{Experimental Design}

To improve the formability, low tensile strength and high ductility are required for alloy steel wires for cold forming. In this study, cold-drawn SCM435 alloy steel wires are investigated. Their chemical composition is shown in Table 1 . The wire coil $(\varnothing 9.0 \mathrm{~mm})$ is previously drawn to a specific size with a section area reduction of about $13.1 \%$.

An intercritical process is used for the spheroidized annealing of the steel wires, in which the wires are heated to an intercritical temperature of $750-780{ }^{\circ} \mathrm{C}$ for $1.5-4.5 \mathrm{~h}$ and then cooled to a lower temperature of $710-745^{\circ} \mathrm{C}$ and held at this temperature for $0.0-6.0 \mathrm{~h}$ before slowly cooling to a temperature of $610-680{ }^{\circ} \mathrm{C}$. The cycle time is about $14-38 \mathrm{~h}$. The experimental tests are conducted in a commercial bell furnace with protective hydrogen atmosphere; the measurements used to monitor the furnace atmosphere are provided with dew point, infrared, and oxygen probes, and a closed-loop atmosphere control system uses hydrogen to minimize the oxide reduction step.

To evaluate the mechanical properties of cold-drawn SCM435 alloy medium-carbon steel wires, seven controllable variables are studied at three levels in this work, as listed in Table 2. The parameters of Level 2 are the original spheroidized annealing process conditions, which were used in Fang Sheng Screw Co., Ltd., Taiwan with a cycle time of $21 \mathrm{~h}$.

The Taguchi method is a combination of mathematical and statistical techniques used in an empirical study that allows simultaneous changes of many factors in a systematic way, ensuring

Table 1

Chemical composition of SCM435 alloy steel wires (wt\%).

\begin{tabular}{ccccccccc}
\hline $\mathrm{C}$ & $\mathrm{P}$ & $\mathrm{Mn}$ & $\mathrm{Si}$ & $\mathrm{Al}$ & $\mathrm{Cu}$ & $\mathrm{Ni}$ & $\mathrm{Cr}$ & $\mathrm{Mo}$ \\
\hline 0.37 & 0.014 & 0.74 & 0.21 & 0.048 & 0.007 & 0.01 & 0.99 & 0.17 \\
\hline
\end{tabular}

Table 2

Experimental factors and their levels for $L_{18}$ orthogonal array.

\begin{tabular}{lccc}
\hline Factor & Level 1 & Level 2 & Level 3 \\
\hline A: Preheating rate $\left({ }^{\circ} \mathrm{C} / \mathrm{h}\right)$ & 100 & 300 & 200 \\
B: Spheroidized annealing temperature $\left({ }^{\circ} \mathrm{C}\right)$ & 750 & 770 & 780 \\
C: Prolonged heating time $(\mathrm{h})$ & 1.5 & 2.5 & 4.5 \\
D: Holding temperature $\left({ }^{\circ} \mathrm{C}\right)$ & 710 & 732 & 745 \\
E: Holding time $(\mathrm{h})$ & 3.0 & 0.0 & 6.0 \\
F: Slow cooling rate $\left({ }^{\circ} \mathrm{C} / \mathrm{h}\right)$ & -6.0 & -8.5 & -15.0 \\
G: Cooling temperature $\left({ }^{\circ} \mathrm{C}\right)$ & 610 & 665 & 680 \\
\hline
\end{tabular}


the reliable and independent study of the factors' effects. The seven variables are assigned to the $L_{18}\left(2^{1} \times 3^{7}\right)$ orthogonal design, ${ }^{(22,23)}$ as shown in Table 3 . This is a highly fractional but efficient design for dealing with seven three-level factors and one two-level factor using only 18 experimental trials. ${ }^{(22,23)}$ The first column is left unassigned.

Three quality characteristics of spheroidized annealed wires, namely, tensile strength, ductility, and hardness, are investigated. Each test trial, including ten specimens that are taken from distinct regions in the bell furnace, is followed by a manufacturing process, and the results are transformed to signal-to-noise $(\mathrm{S} / \mathrm{N})$ ratios. Spheroidizing provides the needed ductility for cold formability. The tensile test is used as a measure of ductility by calculating the elongation of a specimen upon fracture. ${ }^{(24)}$ Tensile tests are conducted on a 20 ton universal testing machine under a constant ram speed of $7 \mathrm{~mm} / \mathrm{min}$ at room temperature. The dimensions of the tensile specimen are $\varnothing 8.39 \mathrm{~mm} \times \mathrm{L} 200 \mathrm{~mm}$. The ductility should be optimized to as high a value as possible and the variation of the ductility should be minimized. Therefore, in terms of the desired characteristics for ductility, the higher the better, and the $\mathrm{S} / \mathrm{N}$ ratio is ${ }^{(22,23)}$

$$
\mathrm{S} / \mathrm{N}=-10 \log \frac{\sum_{i=1}^{n} 1 / y_{i}^{2}}{n},
$$

where $y_{i}$ is the ductility (elongation, $\varepsilon_{f}$ ) of each specimen and $n$ is the test number.

The ductility of the steel wires may be improved through spheroidized annealing, and their strength and hardness, which are obtained from the Rockwell hardness test, may be reduced as well. However, the given strength of the annealed steel wire must be provided for cold forming.

Table 3

$L_{18}$ orthogonal array experimental parameter assignment.

\begin{tabular}{lccccccc}
\hline $\begin{array}{l}\text { Exp. } \\
\text { No. }\end{array}$ & $\begin{array}{c}\text { A: Preheating } \\
\text { rate } \\
\left({ }^{\circ} \mathrm{C} / \mathrm{h}\right)\end{array}$ & $\begin{array}{c}\text { B: Spheroidized } \\
\text { annealing } \\
\text { temperature }\left({ }^{\circ} \mathrm{C}\right)\end{array}$ & $\begin{array}{c}\text { C: Prolonged } \\
\text { heating time } \\
(\mathrm{h})\end{array}$ & $\begin{array}{c}\text { D: Holding } \\
\text { temperature } \\
\left({ }^{\circ} \mathrm{C}\right)\end{array}$ & $\begin{array}{c}\text { E: Holding } \\
\text { time } \\
(\mathrm{h})\end{array}$ & $\begin{array}{c}\text { F: Slow } \\
\text { cooling rate } \\
\left({ }^{\circ} \mathrm{C} / \mathrm{h}\right)\end{array}$ & $\begin{array}{c}\text { G: Cooling } \\
\text { temperature } \\
\left({ }^{\circ} \mathrm{C}\right)\end{array}$ \\
\hline L1 & 100 & 750 & 1.5 & 710 & 3.0 & -6.0 & 610 \\
L2 & 100 & 770 & 2.5 & 732 & 0.0 & -8.5 & 665 \\
L3 & 100 & 780 & 4.5 & 745 & 6.0 & -15.0 & 680 \\
L4 & 300 & 750 & 1.5 & 732 & 0.0 & -15.0 & 680 \\
L5 & 300 & 770 & 2.5 & 745 & 6.0 & -6.0 & 610 \\
L6 & 300 & 780 & 4.5 & 710 & 3.0 & -8.5 & 665 \\
L7 & 200 & 750 & 2.5 & 710 & 6.0 & -8.5 & 680 \\
L8 & 200 & 770 & 4.5 & 732 & 3.0 & -15.0 & 610 \\
L9 & 200 & 780 & 1.5 & 745 & 0.0 & -6.0 & 665 \\
L10 & 100 & 750 & 4.5 & 745 & 0.0 & -8.5 & 610 \\
L11 & 100 & 770 & 1.5 & 710 & 6.0 & -15.0 & 665 \\
L12 & 100 & 780 & 2.5 & 732 & 3.0 & -6.0 & 680 \\
L13 & 300 & 750 & 2.5 & 745 & 3.0 & -15.0 & 665 \\
L14 & 300 & 770 & 4.5 & 710 & 0.0 & -6.0 & 680 \\
L15 & 300 & 780 & 1.5 & 732 & 6.0 & -8.5 & 610 \\
L16 & 200 & 750 & 4.5 & 732 & 6.0 & -6.0 & 665 \\
L17 & 200 & 770 & 1.5 & 745 & 3.0 & -8.5 & 680 \\
L18 & 200 & 780 & 2.5 & 710 & 0.0 & -15.0 & 610 \\
\hline
\end{tabular}


Therefore, the tensile strength of the alloy steel wire is the main quality characteristic. The target value was not specified prior to the experiment, but the variation of the tensile strength should be minimized to obtain annealed wires with an even quality. When a specific target for the response is met (nominal is best), the $\mathrm{S} / \mathrm{N}$ ratio for the response is ${ }^{(22,23)}$

$$
\mathrm{S} / \mathrm{N}=-10 \log \left(\frac{S^{2}}{\mu^{2}}\right)
$$

where $\mu$ and $S$ are the mean and standard deviation of the $n$ tests in each trial, respectively.

ANOVA is an effective method of determining the significant factors and optimal fabrication conditions required to obtain the optimal quality. For the Taguchi method, the experimental error is assessed using ANOVA to inspect the significance of various factors. The nature of the interaction between factors is considered as the experimental error. ${ }^{(22,23)}$ If the effect of a factor in comparison with the experimental error is sufficiently large, it is identified as a significant factor. The confidence level of a factor is assessed using the experimental error to identify the significant factor that affects the material properties of the alloy steel wire.

\section{Results and Discussion}

The microstructures of drawn steel wires are shown in Figs. 1(a) and 1(b) for radial and axial directions, respectively. The drawing trace is very clear along the axial direction, as shown in Fig. 1(b). The tensile strength and hardness are high, about $1005.0 \mathrm{MPa}$ and 105 HRB, respectively, and the ductility is poor, about $0.109^{(25)}$ owing to the heavy plastic work of drawing. Spheroidizing is the process of producing a microstructure in which the cementite

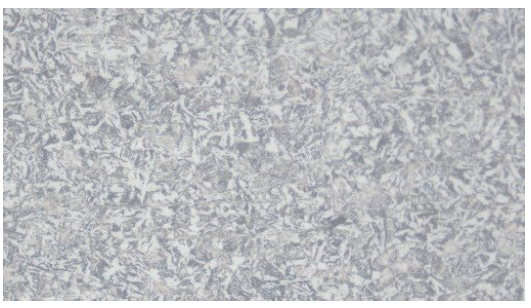

(a)

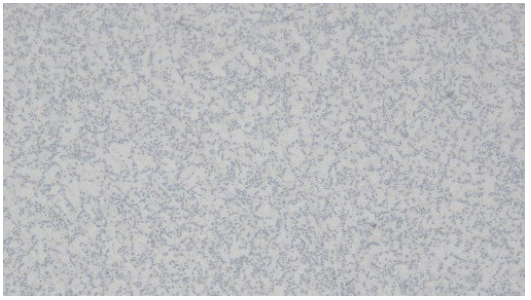

(c)

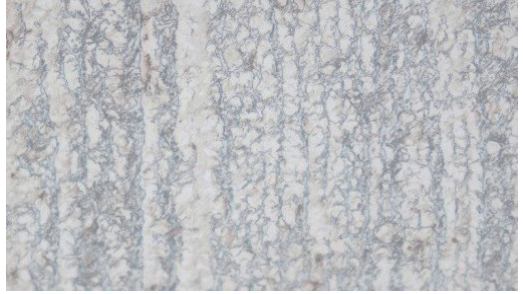

(b)

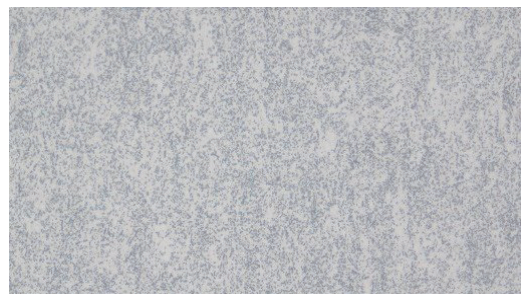

(d)

Fig. 1. (Color online) Microstructures of SCM435 alloy steel wire $(\times 400)$. (a) Drawn (nontreated, radial), (b) drawn (nontreated, axial), (c) intercritical annealed (radial), and (d) intercritical annealed (axial). 
is in a spheroidal distribution, as shown in Figs. 1(c) and 1(d) for radial and axial directions, respectively. The drawing trace along the axial direction almost disappears, as shown in Fig. 1(d). The obtained globular structure improves the formability of the steel wires.

When a wire is fabricated following the original spheroidized annealing process conditions (Level 2 in Table 2), its mean tensile strength and mean hardness are 554.6 MPa and 85.7 HRB, respectively. The tensile strength is about half of that of the nontreated drawn wire and the hardness is also decreased by about $20 \mathrm{HRB}$. The ductility is much improved (about 0.388). However, their variations are large.

The experimental results of the tensile strength and hardness (mean, $\mu$; standard deviation, $S$; and $\mathrm{S} / \mathrm{N}$ ratio) of the spheroidized annealed steel wires are shown in Tables 4 and 5, respectively. The mean tensile strength varies widely from 495.7 (test L3) to $600.4 \mathrm{MPa}$ (test L10), as shown in Table 4. The standard deviation varies from 4.75 (test L3) to $47.60 \mathrm{MPa}$ (test L4), and the result of test L3 is the smallest among the eighteen tests. However, the mean hardness varies from 73.8 (test L3) to 90.4 HRB (test L13), as shown in Table 5, and the mean values of tests L10, L13, and L16 are even larger than the value at the original settings. The standard deviation varies from 0.53 to $5.12 \mathrm{HRB}$, and the result of test L13 is the smallest among the eighteen tests.

As shown in Table 6, the mean ductility varies from 0.288 (test L10) to 0.464 (test L3), and the mean values of tests L3, L5, L6, L8, L9, L14, L15, and L18 are even larger than the value at the original settings. The standard deviation varies from 0.007 (test L13) to 0.072 (test L9). The properties of the spheroidized annealed steel wires clearly vary under various annealing process conditions.

Table 4

Experimental results for tensile strength.

\begin{tabular}{lccccccccccccc}
\hline $\begin{array}{l}\text { Exp. } \\
\text { No. }\end{array}$ & T1 & T2 & T3 & T4 & T5 & T6 & T7 & T8 & T9 & T10 & $\begin{array}{r}\mu \\
\text { (MPa) }\end{array}$ & $S$ & $\begin{array}{r}\text { S/N } \\
\text { ratio }\end{array}$ \\
\hline L1 & 599.8 & 613.1 & 594.4 & 555.0 & 534.3 & 609.5 & 613.7 & 564.6 & 613.8 & 620.4 & 591.9 & 29.86 & 25.9 \\
L2 & 517.3 & 578.2 & 518.8 & 587.7 & 523.5 & 518.0 & 522.4 & 576.9 & 593.5 & 579.2 & 551.6 & 33.66 & 24.3 \\
L3 & 494.4 & 498.6 & 495.6 & 485.8 & 493.0 & 497.1 & 494.3 & 494.3 & 503.5 & 500.4 & 495.7 & 4.75 & 40.4 \\
L4 & 532.5 & 620.2 & 613.7 & 551.8 & 519.7 & 614.2 & 619.8 & 622.3 & 521.2 & 522.2 & 573.8 & 47.60 & 21.6 \\
L5 & 506.4 & 494.1 & 495.7 & 516.8 & 558.0 & 538.0 & 495.7 & 499.4 & 541.1 & 557.2 & 520.2 & 25.98 & 26.0 \\
L6 & 511.1 & 517.8 & 519.8 & 504.8 & 522.7 & 503.8 & 498.1 & 499.8 & 510.6 & 501.4 & 509.0 & 8.78 & 35.3 \\
L7 & 595.2 & 559.0 & 603.0 & 536.1 & 598.5 & 528.4 & 589.9 & 606.6 & 612.2 & 621.6 & 585.0 & 32.40 & 25.1 \\
L8 & 536.8 & 502.8 & 497.1 & 501.9 & 502.3 & 531.0 & 499.6 & 495.7 & 507.9 & 515.6 & 509.1 & 14.30 & 31.0 \\
L9 & 497.4 & 550.6 & 504.8 & 545.6 & 528.2 & 547.5 & 488.0 & 552.6 & 498.4 & 492.2 & 520.5 & 26.84 & 25.8 \\
L10 & 617.0 & 603.2 & 603.6 & 604.4 & 571.0 & 598.7 & 600.2 & 616.7 & 590.9 & 598.5 & 600.4 & 13.05 & 33.3 \\
L11 & 519.1 & 590.1 & 601.2 & 532.5 & 602.7 & 575.2 & 601.6 & 605.4 & 526.1 & 546.6 & 570.0 & 35.28 & 24.2 \\
L12 & 574.8 & 511.8 & 557.4 & 516.4 & 512.9 & 506.5 & 521.2 & 519.7 & 518.0 & 510.9 & 525.0 & 22.50 & 27.4 \\
L13 & 584.4 & 593.0 & 597.4 & 537.5 & 605.9 & 593.2 & 596.9 & 596.5 & 587.6 & 587.8 & 588.0 & 18.79 & 29.9 \\
L14 & 540.3 & 532.4 & 517.2 & 535.5 & 534.7 & 532.4 & 531.7 & 539.6 & 526.3 & 532.6 & 532.3 & 6.64 & 38.1 \\
L15 & 502.7 & 513.6 & 498.9 & 500.4 & 495.5 & 498.8 & 541.5 & 559.0 & 579.2 & 527.6 & 521.7 & 29.33 & 25.0 \\
L16 & 556.1 & 552.3 & 568.7 & 532.8 & 570.7 & 580.2 & 523.1 & 572.1 & 583.5 & 567.1 & 560.7 & 19.78 & 29.1 \\
L17 & 599.1 & 577.4 & 597.0 & 520.2 & 557.0 & 513.2 & 522.9 & 596.2 & 523.5 & 502.2 & 550.9 & 38.75 & 23.1 \\
L18 & 517.4 & 526.8 & 514.9 & 534.8 & 522.9 & 535.2 & 523.9 & 525.1 & 513.0 & 521.8 & 523.6 & 7.48 & 36.9 \\
\hline
\end{tabular}

*Experimental conditions as defined in Table 3. 
Table 5

Experimental results for hardness.

\begin{tabular}{|c|c|c|c|c|c|c|c|c|c|c|c|c|}
\hline $\begin{array}{l}\text { Exp. } \\
\text { No. }\end{array}$ & $\mathrm{T} 1$ & $\mathrm{~T} 2$ & $\mathrm{~T} 3$ & $\mathrm{~T} 4$ & T5 & T6 & $\mathrm{T} 7$ & $\mathrm{~T} 8$ & T9 & $\mathrm{T} 10$ & $\begin{array}{c}\mu \\
\text { (HRB) }\end{array}$ & $S$ \\
\hline L1 & 89.1 & 81.2 & 79.9 & 85.7 & 86.5 & 79.6 & 88.7 & 87.5 & 88.9 & 88.1 & 85.5 & 3.82 \\
\hline L2 & 85.7 & 86.2 & 85.8 & 87.0 & 83.6 & 76.8 & 76.8 & 77.2 & 75.2 & 79.1 & 81.3 & 4.74 \\
\hline L3 & 74.8 & 75.2 & 74.2 & 71.7 & 76.2 & 72.6 & 72.4 & 75.4 & 73.2 & 72.9 & 73.8 & 1.52 \\
\hline L4 & 86.2 & 83.3 & 86.2 & 75.3 & 86.1 & 79.9 & 78.7 & 86.2 & 73.7 & 86.9 & 82.3 & 4.98 \\
\hline L5 & 82.0 & 80.5 & 74.0 & 76.6 & 83.5 & 75.4 & 74.8 & 75.1 & 82.5 & 77.3 & 78.2 & 3.60 \\
\hline L6 & 75.1 & 75.2 & 77.1 & 75.3 & 76.3 & 72.3 & 71.5 & 71.5 & 75.5 & 74.0 & 74.4 & 1.99 \\
\hline L7 & 87.4 & 88.0 & 89.1 & 83.6 & 88.9 & 80.6 & 83.6 & 77.3 & 87.9 & 84.6 & 85.1 & 3.93 \\
\hline L8 & 80.4 & 79.0 & 81.4 & 81.5 & 77.1 & 75.3 & 74.8 & 74.9 & 76.4 & 75.7 & 77.6 & 2.70 \\
\hline L9 & 81.8 & 83.8 & 80.2 & 81.6 & 77.2 & 69.7 & 75.5 & 70.5 & 75.3 & 75.4 & 77.1 & 4.75 \\
\hline L10 & 89.0 & 90.5 & 90.6 & 90.1 & 88.8 & 90.5 & 89.2 & 89.2 & 89.4 & 88.3 & 89.6 & 0.81 \\
\hline L11 & 74.6 & 88.0 & 89.6 & 88.7 & 85.5 & 88.5 & 90.6 & 86.7 & 78.7 & 84.4 & 85.5 & 5.12 \\
\hline L12 & 79.8 & 79.4 & 80.3 & 84.4 & 81.0 & 85.6 & 81.5 & 88.2 & 80.8 & 80.6 & 82.2 & 2.91 \\
\hline L13 & 90.5 & 90.6 & 90.5 & 90.9 & 90.2 & 90.5 & 90.7 & 89.0 & 90.6 & 90.7 & 90.4 & 0.53 \\
\hline L14 & 78.5 & 79.6 & 75.8 & 76.2 & 82.8 & 82.0 & 80.7 & 81.3 & 81.2 & 81.9 & 80.0 & 2.44 \\
\hline L15 & 89.9 & 80.3 & 80.8 & 80.9 & 80.4 & 79.7 & 86.0 & 87.0 & 78.4 & 78.1 & 82.2 & 4.01 \\
\hline L16 & 89.5 & 90.3 & 89.4 & 89.2 & 87.0 & 89.7 & 84.7 & 90.1 & 87.9 & 85.5 & 88.3 & 1.98 \\
\hline L17 & 91.0 & 90.2 & 82.7 & 80.9 & 83.6 & 87.3 & 80.0 & 81.7 & 84.8 & 89.0 & 85.1 & 4.00 \\
\hline L18 & 77.8 & 79.0 & 82.3 & 82.8 & 82.1 & 80.6 & 80.3 & 81.0 & 81.4 & 84.4 & 81.2 & 1.90 \\
\hline
\end{tabular}

*Experimental conditions as defined in Table 3.

Table 6

Experimental results for ductility.

\begin{tabular}{lccccccccccccr}
\hline $\begin{array}{l}\text { Exp. } \\
\text { No. }\end{array}$ & T1 & T2 & T3 & T4 & T5 & T6 & T7 & T8 & T9 & T10 & $\mu$ & $S$ & $\begin{array}{r}\text { S/N } \\
\text { ratio }\end{array}$ \\
\hline L1 & 0.294 & 0.294 & 0.326 & 0.305 & 0.376 & 0.302 & 0.280 & 0.247 & 0.290 & 0.294 & 0.301 & 0.033 & -10.6 \\
L2 & 0.429 & 0.281 & 0.398 & 0.282 & 0.397 & 0.441 & 0.388 & 0.278 & 0.297 & 0.280 & 0.347 & 0.069 & -9.6 \\
L3 & 0.480 & 0.466 & 0.470 & 0.477 & 0.445 & 0.454 & 0.484 & 0.475 & 0.458 & 0.432 & 0.464 & 0.017 & -6.7 \\
L4 & 0.320 & 0.303 & 0.283 & 0.333 & 0.379 & 0.285 & 0.284 & 0.289 & 0.393 & 0.396 & 0.326 & 0.046 & -9.9 \\
L5 & 0.414 & 0.464 & 0.455 & 0.389 & 0.365 & 0.366 & 0.463 & 0.467 & 0.378 & 0.367 & 0.413 & 0.045 & -7.8 \\
L6 & 0.463 & 0.445 & 0.439 & 0.450 & 0.453 & 0.431 & 0.463 & 0.453 & 0.435 & 0.459 & 0.449 & 0.011 & -7.0 \\
L7 & 0.290 & 0.304 & 0.267 & 0.371 & 0.290 & 0.315 & 0.301 & 0.282 & 0.297 & 0.299 & 0.302 & 0.028 & -10.5 \\
L8 & 0.361 & 0.462 & 0.446 & 0.477 & 0.412 & 0.378 & 0.422 & 0.462 & 0.419 & 0.369 & 0.421 & 0.041 & -7.6 \\
L9 & 0.484 & 0.323 & 0.446 & 0.357 & 0.354 & 0.349 & 0.482 & 0.309 & 0.454 & 0.487 & 0.404 & 0.072 & -8.2 \\
L10 & 0.289 & 0.276 & 0.294 & 0.291 & 0.264 & 0.280 & 0.295 & 0.304 & 0.303 & 0.287 & 0.288 & 0.012 & -10.8 \\
L11 & 0.396 & 0.313 & 0.312 & 0.369 & 0.312 & 0.299 & 0.304 & 0.317 & 0.420 & 0.387 & 0.343 & 0.045 & -9.5 \\
L12 & 0.337 & 0.416 & 0.330 & 0.405 & 0.386 & 0.398 & 0.372 & 0.369 & 0.403 & 0.410 & 0.383 & 0.030 & -8.4 \\
L13 & 0.293 & 0.284 & 0.290 & 0.287 & 0.299 & 0.291 & 0.300 & 0.288 & 0.304 & 0.286 & 0.292 & 0.007 & -10.7 \\
L14 & 0.406 & 0.408 & 0.425 & 0.428 & 0.412 & 0.403 & 0.418 & 0.391 & 0.421 & 0.438 & 0.415 & 0.014 & -7.7 \\
L15 & 0.444 & 0.419 & 0.456 & 0.405 & 0.444 & 0.455 & 0.362 & 0.323 & 0.333 & 0.340 & 0.398 & 0.054 & -8.2 \\
L16 & 0.322 & 0.255 & 0.319 & 0.268 & 0.270 & 0.315 & 0.356 & 0.285 & 0.323 & 0.338 & 0.305 & 0.034 & -10.5 \\
L17 & 0.298 & 0.296 & 0.351 & 0.355 & 0.324 & 0.369 & 0.387 & 0.293 & 0.352 & 0.391 & 0.342 & 0.037 & -9.5 \\
L18 & 0.420 & 0.390 & 0.420 & 0.398 & 0.401 & 0.386 & 0.420 & 0.395 & 0.398 & 0.426 & 0.405 & 0.015 & -7.9 \\
\hline
\end{tabular}

*Experimental conditions as defined in Table 3. 


\subsection{Tensile strength}

To obtain the optimal quality, ANOVA is carried out to determine significant factors and optimal fabrication conditions. The contribution and confidence level of each factor constructed in Table 7 could identify the significant factor affecting the tensile strength of the wires. The contribution of a factor is the percentage of the sum of squares $(S S)$, that is, the percentage of the factor variance to the total quality loss. ${ }^{(23)}$

The effect of a factor may be pooled to error if its confidence level or contribution is relatively small. It is obvious from the ANOVA table that the contribution of prolonged heating time (C) is $59.93 \%$ of the total variation, which clearly indicates that the prolonged heating time is the largest contributor to the variability of the experimental results. The contributions of spheroidized annealing temperature (B, 12.91\%) and holding temperature $(\mathrm{D}, 12.36 \%)$ are the second and third largest, respectively, which are almost the same, as shown in Table 7. However, the other four factors are not significant for the $\mathrm{S} / \mathrm{N}$ ratio since their contributions are relatively small. With the pooling of errors from the nonsignificant factors (A, E, F, and G), the error for the $\mathrm{S} / \mathrm{N}$ ratio is estimated, ${ }^{(23)}$ and then the confidence levels are $96.83,99.99$, and $96.45 \%$, respectively, for spheroidized annealing temperature (B), prolonged heating time (C), and holding temperature (D). That is, the three factors, especially the prolonged heating time, significantly affect the tensile strength of the alloy steel wire with a confidence level of more than $95.0 \%$.

Table 7

ANOVA results of $\mathrm{S} / \mathrm{N}$ ratio for tensile strength.

\begin{tabular}{|c|c|c|c|c|c|c|}
\hline Factor & $S S$ & DOF & Var. & \multicolumn{2}{|c|}{ Contribution } & \\
\hline $\mathrm{A}$ & 2.50 & 2 & 1.25 & \multicolumn{2}{|c|}{$0.47 \%$} & \\
\hline B & 68.97 & 2 & 34.49 & \multicolumn{2}{|l|}{$12.91 \%$} & \\
\hline $\mathrm{C}$ & 320.11 & 2 & 160.05 & \multicolumn{2}{|l|}{$59.93 \%$} & \\
\hline $\mathrm{D}$ & 66.00 & 2 & 33.00 & \multicolumn{2}{|l|}{$12.36 \%$} & \\
\hline $\mathrm{E}$ & 9.16 & 2 & 4.58 & \multicolumn{2}{|l|}{$1.71 \%$} & \\
\hline $\mathrm{F}$ & 27.90 & 2 & 13.95 & \multicolumn{2}{|l|}{$5.22 \%$} & \\
\hline G & 8.49 & 2 & 4.25 & \multicolumn{2}{|l|}{$1.59 \%$} & \\
\hline Others & 30.97 & 3 & 10.32 & \multicolumn{2}{|l|}{$5.80 \%$} & \\
\hline Total & 534.10 & 17 & - & \multicolumn{2}{|l|}{$100.00 \%$} & \\
\hline \multicolumn{7}{|c|}{ Pooling of errors } \\
\hline Factor & $S S$ & DOF & Var. & $F$ & Confidence & Significance \\
\hline$\overline{\mathrm{A}}$ & & & & \multicolumn{3}{|c|}{ Pooled } \\
\hline B & 68.97 & 2 & 34.49 & 4.80 & $96.83 \%$ & Yes \\
\hline $\mathrm{C}$ & 320.11 & 2 & 160.05 & 22.28 & $99.99 \%$ & Yes \\
\hline $\mathrm{D}$ & 66.00 & 2 & 33.00 & 4.59 & $96.45 \%$ & Yes \\
\hline $\mathrm{E}$ & & & & \multicolumn{3}{|c|}{ Pooled } \\
\hline $\mathrm{F}$ & & & & \multicolumn{3}{|c|}{ Pooled } \\
\hline G & & & & \multicolumn{3}{|c|}{ Pooled } \\
\hline Others & & & & \multicolumn{3}{|c|}{ Pooled } \\
\hline Error & 79.02 & 11 & 7.18 & \multicolumn{2}{|c|}{$S_{\text {exp }}=2.68$} & \\
\hline Total & 534.10 & 17 & & \multicolumn{3}{|c|}{ *Note: At least $95.00 \%$ confidence } \\
\hline
\end{tabular}

SS: sum of squares; DOF: degree of freedom; Var.: variance; F: F-ratio; $S_{\text {exp }}$ : experimental error. 


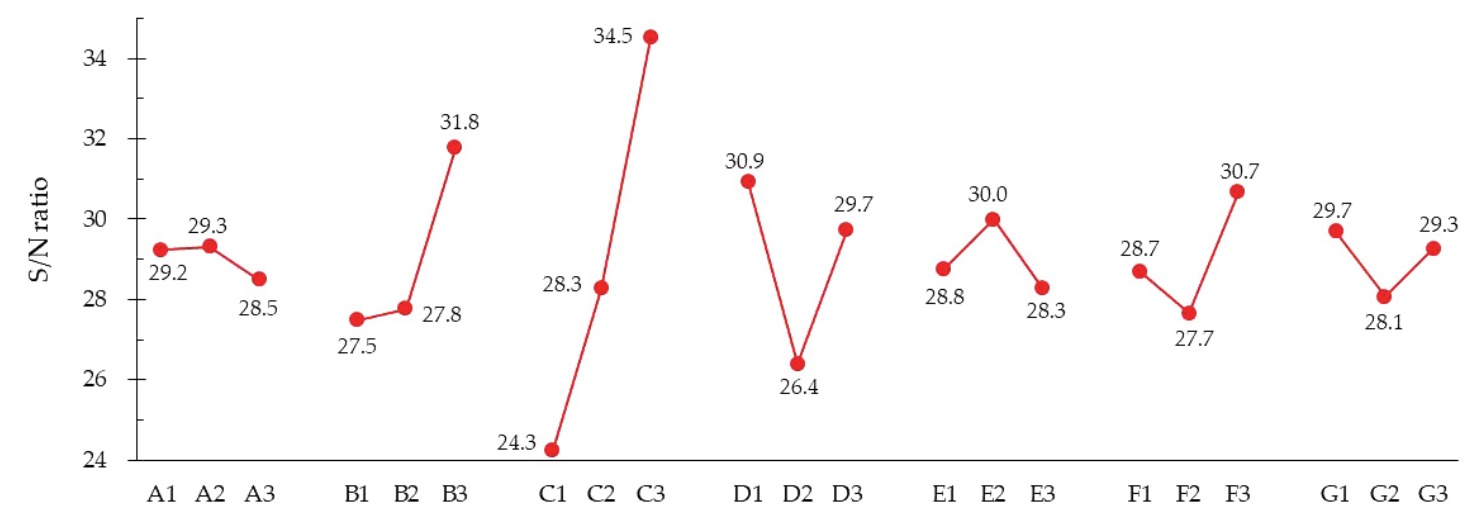

Fig. 2. (Color online) Factor response diagram for tensile strength.

Figure 2 illustrates the factor response diagram and the level averages of seven factors with respect to the $\mathrm{S} / \mathrm{N}$ ratio. For each factor, the effect is indicated by a range of level averages, and the maximum level average is considered the optimal level. ${ }^{(22,23)}$ It is clearly revealed that, for the seven factors, the original levels (Level 2) are not the optimal fabrication parameters for obtaining the target tensile strength.

For the significant factors of spheroidized annealing temperature (B), prolonged heating time (C), and holding temperature (D), Level 3 for the spheroidized annealing temperature $\left(780{ }^{\circ} \mathrm{C}\right.$, B3), Level 3 for the prolonged heating time (4.5 h, C3), and Level 1 for the holding temperature $\left(710{ }^{\circ} \mathrm{C}, \mathrm{D} 1\right)$ are evidently the optimal levels, as shown in Fig. 2. It is observed that the response is almost linear with the prolonged heating time, but is not linear with the holding temperature. For the spheroidized annealing temperature, the response of the optimal level is much more effective than the other two levels.

For the other four nonsignificant factors, the optimal levels are respectively Level 2 for the preheating rate $\left(300^{\circ} \mathrm{C} / \mathrm{h}, \mathrm{A} 2\right)$, Level 2 for the holding time $(0.0 \mathrm{~h}, \mathrm{E} 2)$, Level 3 for the slow cooling rate $\left(-15^{\circ} \mathrm{C} / \mathrm{h}, \mathrm{F} 3\right)$, and Level 1 for the cooling temperature $\left(610^{\circ} \mathrm{C}, \mathrm{G} 1\right)$.

\subsection{Ductility}

The ANOVA results of the $\mathrm{S} / \mathrm{N}$ ratio for the ductility of the annealed steel wires are shown in Table 8. It is evident from Table 8 that the largest contributor to the variability of the experimental results is predominantly the spheroidized annealing temperature ( $\mathrm{B}, 74.38 \%$ ). The contribution of the prolonged heating time (C) is $9.67 \%$, which is the second largest contribution.

However, the preheating rate (A), holding temperature (D), holding time (E), slow cooling rate $(\mathrm{F})$, and cooling temperature $(\mathrm{G})$ are not significant factors because their contributions are relatively small. With the pooling of errors from the nonsignificant factors (A, D, E, F, and G), the confidence levels are 100.00 and $95.42 \%$ for the spheroidized annealing temperature (B) and prolonged heating time $(\mathrm{C})$, respectively. That is, the ductility of the steel wires is significantly affected by the spheroidized annealing temperature and prolonged heating time, with a confidence level of more than $95.00 \%$. 
Table 8

ANOVA results of $\mathrm{S} / \mathrm{N}$ ratio for ductility.

\begin{tabular}{lrrrr}
\hline Factor & \multicolumn{1}{c}{$S S$} & DOF & \multicolumn{1}{c}{ Var. } & Contribution \\
\hline A & 1.63 & 2 & 0.815 & $5.07 \%$ \\
B & 23.92 & 2 & 11.960 & $74.38 \%$ \\
C & 3.11 & 2 & 1.556 & $9.67 \%$ \\
D & 0.14 & 2 & 0.072 & $0.45 \%$ \\
E & 0.08 & 2 & 0.042 & $0.26 \%$ \\
F & 0.99 & 2 & 0.497 & $3.09 \%$ \\
G & 0.81 & 2 & 0.406 & $2.53 \%$ \\
Others & 1.46 & 3 & 0.487 & $4.54 \%$ \\
Total & 32.16 & 17 & - & $100.00 \%$
\end{tabular}

\begin{tabular}{|c|c|c|c|c|c|c|}
\hline \multicolumn{7}{|c|}{ Pooling of errors } \\
\hline Factor & $S S$ & DOF & Var. & $F$ & Confidence & Significance \\
\hline$\overline{\mathrm{A}}$ & & & & \multicolumn{3}{|c|}{ Pooled } \\
\hline $\mathrm{B}$ & 23.92 & 2 & 11.960 & 30.33 & $100.00 \%$ & Yes \\
\hline $\mathrm{C}$ & 3.11 & 2 & 1.556 & 3.94 & $95.42 \%$ & Yes \\
\hline $\mathrm{D}$ & & & & \multicolumn{3}{|c|}{ Pooled } \\
\hline $\mathrm{E}$ & & & & \multicolumn{3}{|c|}{ Pooled } \\
\hline $\mathrm{F}$ & & & & \multicolumn{3}{|c|}{ Pooled } \\
\hline G & & & & \multicolumn{3}{|c|}{ Pooled } \\
\hline Others & & & & \multicolumn{3}{|c|}{ Pooled } \\
\hline Error & 5.13 & 13 & 0.394 & \multicolumn{3}{|c|}{$S_{\text {exp }}=0.63$} \\
\hline Total & 32.16 & 17 & & \multicolumn{3}{|c|}{${ }^{*}$ Note: At least $95.00 \%$ confidence } \\
\hline
\end{tabular}

SS: sum of squares; DOF: degree of freedom; Var.: variance; F: F-ratio; $S_{\text {exp }}$ : experimental error.

The factor response diagram and the level averages of the seven factors with respect to the $\mathrm{S} / \mathrm{N}$ ratio are illustrated in Fig. 3. It is clearly revealed that, for the seven factors, the original levels (Level 2) are not either the optimal fabrication parameters for obtaining the higher ductility and that most of the effects are smaller than the effects of tensile strength, as shown in Fig. 2. For the two significant factors of spheroidized annealing temperature (B) and prolonged heating time (C), the optimal levels are Level 3 for the spheroidized annealing temperature (780 $\left.{ }^{\circ} \mathrm{C}, \mathrm{B} 3\right)$ and Level 3 for prolonged heating time (4.5 h, C3), as shown in Fig. 3.

The effects of the other five factors are relatively small. The optimal levels are respectively Level 2 for the preheating rate $\left(300{ }^{\circ} \mathrm{C} / \mathrm{h}, \mathrm{A} 2\right)$, Level 1 for holding temperature $\left(710^{\circ} \mathrm{C}, \mathrm{D} 1\right)$, Level 3 for the holding time $(6.0 \mathrm{~h}, \mathrm{E} 3)$, Level 3 for the slow cooling rate $\left(-15.0^{\circ} \mathrm{C} / \mathrm{h}, \mathrm{F} 3\right)$, and Level 3 for the cooling temperature $\left(680^{\circ} \mathrm{C}, \mathrm{G} 3\right)$.

With the results of the optimal quality characteristics of tensile strength and ductility, the optimal conditions are shown in Table 9. The spheroidized annealing temperature (B) and prolonged heating time (C) are clearly significant for both the tensile strength and the ductility and at the same levels. Since the tensile strength of the steel wires is the main quality characteristic and has higher effects, the optimal levels are thus determined as Level 3 for the spheroidized annealing temperature $\left(780^{\circ} \mathrm{C}, \mathrm{B} 3\right)$ and Level 3 for the prolonged heating time (4.5 $\mathrm{h}, \mathrm{C} 3$ ). The holding temperature (D) is significant for the tensile strength but not for ductility. Thus, Level 1 for the holding temperature $\left(710^{\circ} \mathrm{C}\right.$, D1) is then chosen as the optimal level. 


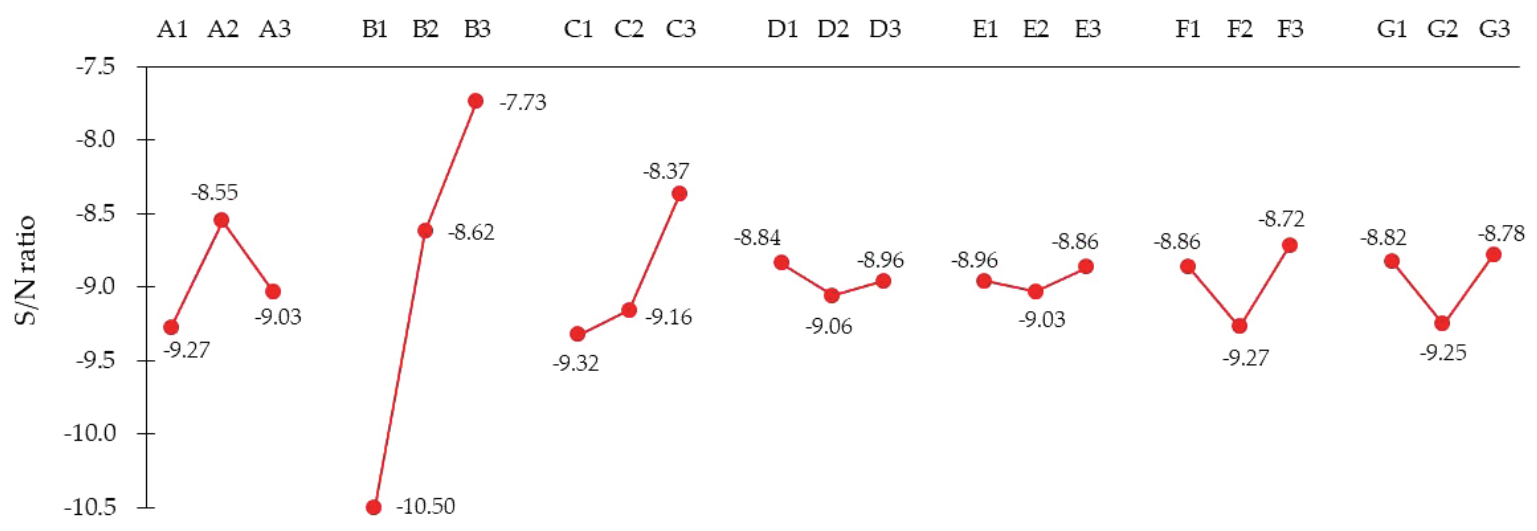

Fig. 3. (Color online) Factor response diagram for ductility.

Table 9

Optimal conditions for spheroidized annealing.

\begin{tabular}{lccc}
\hline Factor & Tensile strength & Ductility & Optimal \\
\hline A: Preheating rate $\left({ }^{\circ} \mathrm{C} / \mathrm{h}\right)$ & $\mathrm{A} 2$ & $\mathrm{~A} 2$ & $\mathrm{~A} 2$ \\
B: Spheroidized annealing temperature $\left({ }^{\circ} \mathrm{C}\right)$ & $\mathrm{B}^{*}$ & $\mathrm{~B} 3$ & \\
C: Prolonged heating time $(\mathrm{h})$ & $\mathrm{C}{ }^{*}$ & $\mathrm{C} 3$ & $\mathrm{~B} 3$ \\
D: Holding temperature $\left({ }^{*} \mathrm{C}\right)$ & $\mathrm{D}{ }^{*}$ & $\mathrm{D} 1$ & $\mathrm{C} 3$ \\
E: Holding time $(\mathrm{h})$ & $\mathrm{E} 2$ & $\mathrm{E} 3$ & $\mathrm{D} 1$ \\
F: Slow cooling rate $\left({ }^{\circ} \mathrm{C} / \mathrm{h}\right)$ & $\mathrm{F} 3$ & $\mathrm{~F} 3$ & $\mathrm{E} 2$ \\
G: Cooling temperature $\left({ }^{\circ} \mathrm{C}\right)$ & $\mathrm{G} 1$ & $\mathrm{G} 3$ & $\mathrm{~F} 3$ \\
\hline
\end{tabular}

${ }^{*}$ Significant factor.

The other four factors are not significant either for the tensile strength or ductility, as shown in Table 9; thus, Level 2 for the preheating rate $\left(300^{\circ} \mathrm{C} / \mathrm{h}, \mathrm{A} 2\right)$, Level 2 for the holding time $(0.0 \mathrm{~h}$, E2), Level 3 for the slow cooling rate $\left(-15.0^{\circ} \mathrm{C} / \mathrm{h}, \mathrm{F} 3\right)$, and Level 1 for the cooling temperature (610 $\left.{ }^{\circ} \mathrm{C}, \mathrm{G} 1\right)$ are determined.

\subsection{Confirmation experiments}

To verify the predicted results, the alloy steel wires are fabricated using the optimal levels A2, B3, C3, D1, E2, F3, and G1, as described in Table 9, which takes a cycle time of $20.25 \mathrm{~h}$. Figures 4-6 show the nontreated, original (using Level $2 \mathrm{~s}$ in Table 2), and optimal probability distributions, respectively, for the tensile strength, hardness, and ductility of cold-drawn SCM435 alloy steel wires.

It is observed that, through spheroidized annealing treatment with the original settings, the mean tensile strength, as compared with the nontreated results, is decreased substantially by about $450 \mathrm{MPa}$, as shown in Fig. 4, and the deviations are almost the same. The mean hardness is also decreased by about $19 \mathrm{HRB}$, as shown in Fig. 5, but the deviation is clearly increased by about $180 \%$. However, the mean ductility is simultaneously increased by about 0.28 , as shown in Fig. 6, but the deviation is substantially increased by about 13.6 times. 


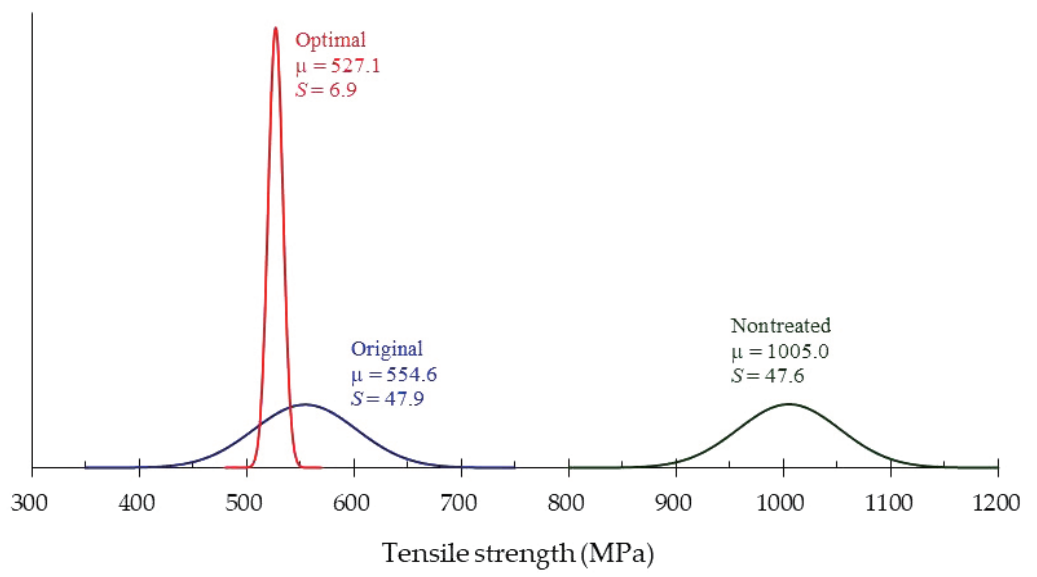

Fig. 4. (Color online) Probability distribution diagram for tensile strength.

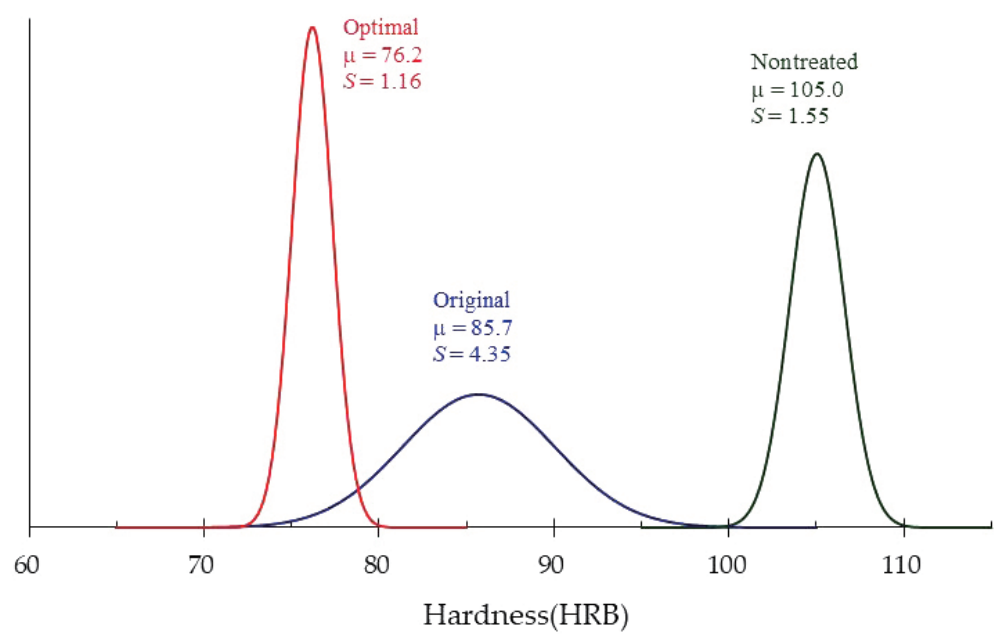

Fig. 5. (Color online) Probability distribution diagram for hardness.

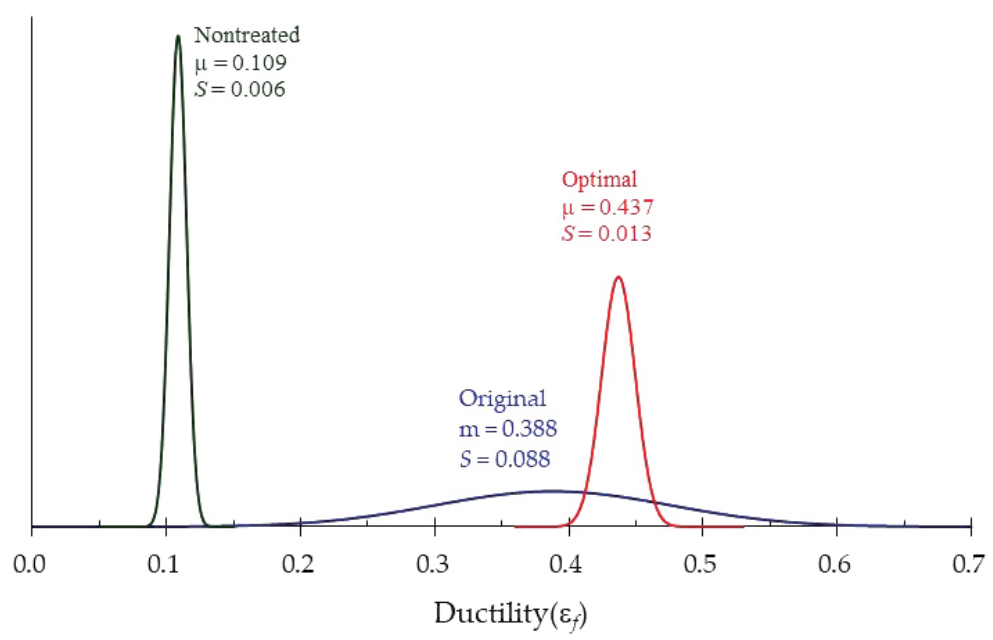

Fig. 6. (Color online) Probability distribution diagram for ductility. 
Compared with the original results, the optimal mean tensile strength of $527.1 \mathrm{MPa}$ is not only decreased by about $27 \mathrm{MPa}$, as shown in Fig. 4, but also the deviation is substantially decreased by about $86 \%$. The optimal mean hardness is also decreased by about $9 \mathrm{HRB}$, as shown in Fig. 5, and the deviation is clearly decreased by about $73 \%$ as well. The optimal mean ductility of 0.437 , as shown in Fig. 6 , is slightly increased by about 0.049 , whereas the deviation is substantially decreased by about $85 \%$ as compared with the original result. The scatter of these individual values is significantly reduced. Furthermore, the cycle time is reduced. The new parameter settings evidently improve the performance measures, such as strength, hardness, and ductility, over their values at the original settings, as well as the quality of the spheroidized annealed alloy steel wires. Therefore, the formability of the cold-drawn SCM435 alloy steel wires is effectively improved.

\section{Materials and Methods}

In this study, the wires are spheroidized-annealed after drawing the SCM435 alloy steel wire coil $(\varnothing 9.0 \mathrm{~mm})$ to a specific size $(\varnothing 8.39 \mathrm{~mm})$ with a section area reduction of about $13.1 \%$. The steel wire coil is manufactured ( $\varnothing 9.0 \mathrm{~mm}$, Al-killed) by China Steel Corporation, Kaohsiung, Taiwan. Its chemical composition is listed in Table 1.

The alloy steel wire coil is spheroidized-annealed by an intercritical annealing procedure, as shown in Fig. 7, in a commercial A210 bell furnace (Rad-Con Inc., Cleveland, OH, USA) with protective hydrogen atmosphere. The temperatures are measured using thermocouples and precisely controlled within $\pm 2{ }^{\circ} \mathrm{C}$. By using a zirconium oxide sensor in conjunction with a proprietary algorithm known as annealing process equilibrium, a closed-loop atmosphere control system accurately and repeatedly senses and adjusts cycle after cycle to determine the optimal atmosphere flow settings.

The Taguchi method allows the changing of many factors at the same time in a systematic way. The orthogonal array table $L_{18}$ is used as an experimental design for the factors, ${ }^{(23)}$ as listed in Table 3.

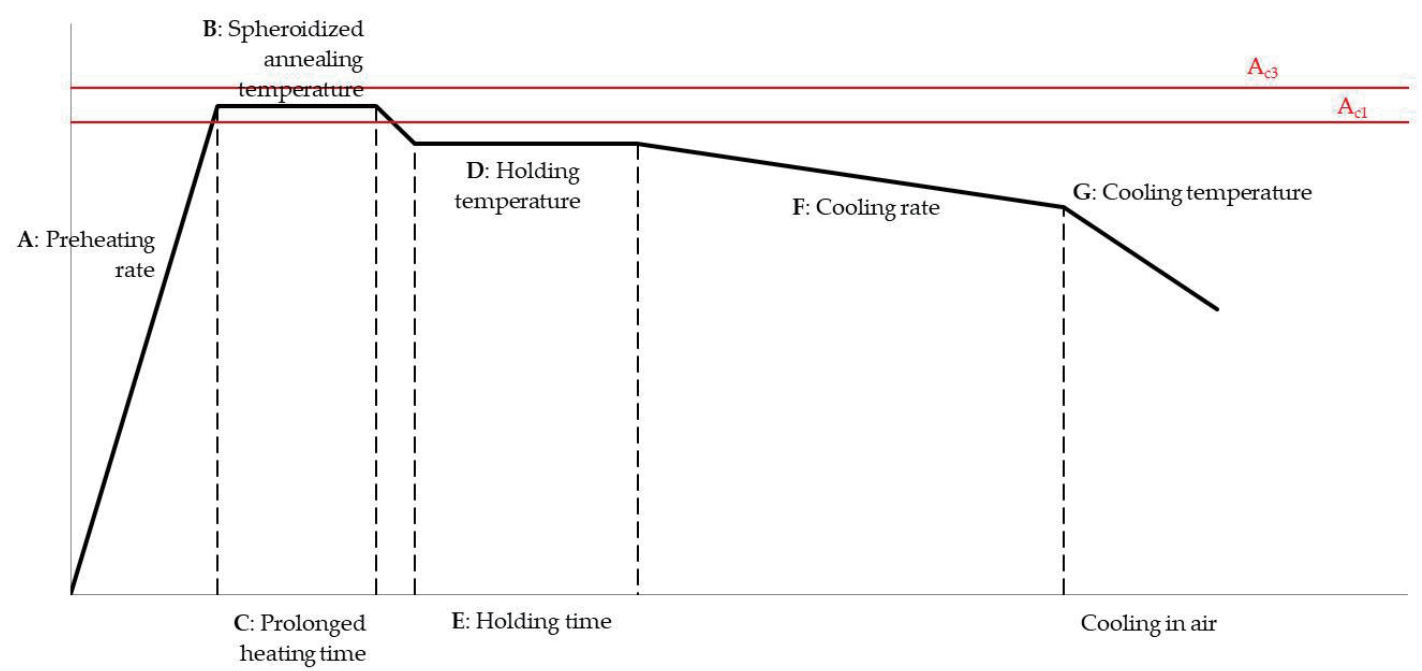

Fig. 7. (Color online) Intercritical annealing procedure. 


\section{Conclusions}

The Taguchi method along with ANOVA is used to obtain optimal intercritical annealing conditions to improve the mechanical properties of cold-drawn SCM435 alloy steel wires. The annealing experiments are conducted in a commercial bell furnace with hydrogen atmosphere, in which temperatures are precisely controlled within $\pm 2{ }^{\circ} \mathrm{C}$ and a closed-loop atmosphere control system is applied to determine the optimal atmosphere flow settings by using a zirconium oxide sensor in conjunction with annealing process equilibrium. The spheroidized annealing qualities of alloy steel wires are affected by various factors, such as preheating rate, spheroidized annealing temperature, prolonged heating time, holding temperature and time, and slow cooling rate and temperature. The spheroidized annealing conditions affect the quality characteristics of alloy steel wires, such as tensile strength, hardness, and ductility. The variation of the properties should be minimized to obtain annealed wires with an even quality.

It is experimentally revealed that the spheroidized annealing temperature (B), prolonged heating time (C), and holding temperature (D) are significant factors; the determined levels are Level 3 for spheroidized annealing temperature $\left(780^{\circ} \mathrm{C}\right.$, B3), Level 3 for prolonged heating time $(4.5 \mathrm{~h}, \mathrm{C} 3)$, Level 1 for holding temperature $\left(710^{\circ} \mathrm{C}, \mathrm{D} 1\right)$, Level 2 for the preheating rate $\left(300{ }^{\circ} \mathrm{C} / \mathrm{h}\right.$, A2), Level 2 for holding time $(0.0 \mathrm{~h}, \mathrm{E} 2)$, Level 3 for slow cooling rate $\left(-15.0{ }^{\circ} \mathrm{C} /\right.$ $\mathrm{h}, \mathrm{F} 3)$, and Level 1 for cooling temperature $\left(610^{\circ} \mathrm{C}, \mathrm{G} 1\right)$. Therefore, the optimal mean tensile strength of $527.1 \mathrm{MPa}$, optimal mean hardness of $76.2 \mathrm{HRB}$, and optimal mean ductility of 0.437 are obtained with a shorter processing time. The variations of the properties are significantly reduced to achieve a much even quality for annealed wires. The new spheroidizing parameter settings evidently improve the performance measures over their values at the original settings. The formability of cold-drawn SCM435 alloy steel wires is substantially improved.

\section{Acknowledgments}

The authors would like to acknowledge the support of Fang Sheng Screw Co., Ltd., Kaohsiung, Taiwan, for providing the materials and apparatus to carry out the spheroidized annealing experimental work.

\section{References}

1 J. Dhers, B. Thivard, and A. Genta: Wire J. Int. 25 (1992) 73.

2 B. L. Bramfitt and A. K. Hingwe: Annealing of Steel (ASM International, Ohio, 1991).

3 N. V. Luzginova, L. Zhao, and J. Sietsma: Metall. Mater. Trans. A 39 (2008) 513. https://doi.org/10.1007/ s11661-007-9403-3

4 P. Johnson: Furnace Atmospheres (ASM International, Ohio, 1991).

5 W. Scheuermann, D. Böing, P. Wittler, M. A. Bock, and T. Bovalina: MPT Metall. Plant Technol. 18 (1995) 90.

6 C. J. Messina: Fastener Technol. Int. XXXIV (2011) 26.

7 A. Saboonchi and S. Hassanpour: Exp. Heat Transfer 21 (2008) 220. https://doi.org/10.1080/08916150802072867

8 S. C. Ikpeseni, B. O. Onyekpe, and H. Ovri: Nigerian J. Technol. 34 (2015) 499. https://doi.org/10.4314/njt. v34i3.11

9 J. C. Mun and C.-G. Kang: Int. J. Precis. Eng. Manuf. 16 (2015) 1299. https://doi.org/10.1007/s12541-0150169-Z

10 C.-C. Yang and C.-L. Liu: Materials 9 (2016) 693. https://doi.org/10.3390/ma9080693 
11 C.-C. Yang and S.-T. Wang: Sens. Mater. 30 (2018) 503. https://doi.org/10.18494/SAM.2018.1755

12 D. Hernāndez-Silva, R. D. Morales, and J. G. Cabaňas-Moreno: ISIJ Int. 32 (1992) 1297. https://doi. org/10.2355/isijinternational.32.1297

13 J. M. O’Brien and W. F. Hosford: Metall. Mater. Trans. A 33 (2002) 1255. https://doi.org/10.1007/s11661-0020226-y

14 E. Karadeniz: Mater. Des. 29 (2008) 251. https://doi.org/10.1016/j.matdes.2006.11.015

15 Y. G. Ko, S. Namgung, D. H. Shin, I. H. Son, K. H. Rhee, and D.-L. Lee: J. Mater. Sci. 45 (2010) 4866. https:// doi.org/10.1007/s10853-010-4587-0

16 A. Kamyabi-Gol and M. Sheikh-Amiri: J. Iron. Steel Res. Int. 17 (2010) 45. https://doi.org/10.1016/S1006$706 \times(10) 60085-4$

17 J. Arruabarrena, P. Uranga, B. López, and J. M. Rodríguez-Ibabe: Proc. Mater. Sci. Technol. Conf. (Columbus, Ohio, 2011) 698-705.

18 C. Ji, L. Wang, and M.-Y. Zhu: J. Iron. Steel Res. Int. 22 (2015) 1031. https://doi.org/10.1016/S1006706X(15)30108-4

19 H. S. Joo, S. K. Hwang, H. M. Baek, Y.-T. Im, I.-H. Son, and C. M. Bae: J. Mater. Process. Technol. 216 (2015) 348. https://doi.org/10.1016/j.jmatprotec.2014.10.001

20 M. Okonogi and K. Yamazaki: Nippon Steel \& Sumitomo Metal Technical Report 116 (2017) 65.

21 C.-C. Yang and N.-H. Lu: Adv. Mater. 7 (2018) 144. https://doi.org/10.11648/j.am.20180704.17

22 N. Logothetis: Managing for Total Quality: From Deming to Taguchi and SPC (Prentice Hall International: London, UK, 1992).

23 H.-H. Lee: Taguchi Methods: Principles and Practices of Quality Design (Gau Lih Book, New Taipei City, 2008).

24 P. F. Ostwald and J. Munoz: Manufacturing Processes and Systems (Wiley, New York, 1997).

25 C.-C. Yang and N.-H. Lu: Mater. Sci. Appl. 10 (2019) 677. https://doi.org/10.4236/msa.2019.1011048

\section{About the Authors}

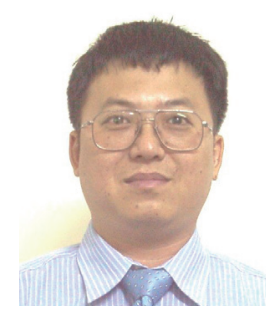

Chih-Cheng Yang received his B.S. degree from Tatung Institute of Technology, Taiwan, in 1985, his M.S. degree from National Cheng Kung University, Taiwan, in 1991, and his Ph.D. degree from Liverpool University, UK, in 1997. From 2000 to 2016, he was an assistant professor at Kao Yuan University, Taiwan. Since 2017, he has been an associate professor at Kao Yuan University. His research interests are in materials, manufacturing, and optimum design of engineering. (t30043@cc.kyu.edu.tw)

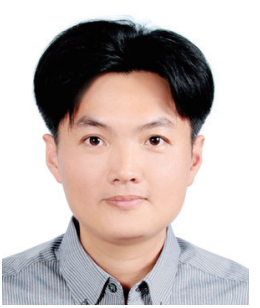

Nan-Hua Lu received his associate degree from Chia Nan University, Taiwan, in 1998 and his M.S. degree from Kao Yuan University, Taiwan, in 2019. Since 2000, he was one of the entrepreneurs who conducted their business with Nahua Electrical Company. Since 2016, he has been working as an electrical manager at American RAD-CON Co., Ltd. His research interests are in materials and annealing of fasteners. (nahua@Livemail.tw) 